\title{
A Solanum incanum extract (SR-T100) regresses vulvar condyloma acuminatum and induces distinct autophagic and apoptotic responses in different types of HPV-infected cells
}

Yeong-Chang Chen ${ }^{1}$, Meng-Ru Shen ${ }^{1}$, Ai-Li Shiau², Hamm-Ming Sheu ${ }^{3}$, Yu-Lin Liang ${ }^{1}$ and Keng-Fu Hsu*

\begin{abstract}
Background: The Solanum species have been used for the treatment of warts, tumor and cancer in folk medicine. The S. incanum extract is an important traditional Chinese medicine in Taiwan since 1973. The purpose of the present study was to evaluate the efficacy and safety of Solanum incanum (synonym: Solanum undatum) extract (SR-T100), a water-soluble product primarily composed of alkaloid solamargine, for the treatment of human condyloma and to study the possible underlying anti-condyloma mechanisms.
\end{abstract}

Methods: We conducted a pilot study to test the effectiveness and side effects of SR-T100 gel (2.3\% solamargine in Solanum incanum plant extract) for the treatment of external genital warts. We produced different types of human papillomavirus (HPV) E6/E7-infected cells by lentiviral technology and studied the differences in apoptosis and autophagy between these cells under the treatment of SR-T100.

Results: Nineteen (73\%) of 26 patients using the SR-T100 gel exhibited a response, and 16 (61.5\%) patients achieved total clearance. Only one patient showed severe (grade 3-4) skin-related side effects. SR-T100 induced mitochondriadependent apoptosis in HPV-infected cells. Cells expressing the high-risk HPV E6/E7 type were resistant to SR-T100induced apoptosis. SR-T100 induced a greater autophagic response in HPV 16, 18-E6/E7 cells than in HPV 6b, 11-E6/E7 cells. Autophagy inhibition enhanced SR-T100-induced apoptosis in HPV 16, 18-E6/E7 cells, whereas apoptosis inhibition enhanced SR-T100-induced autophagy in HPV 6b, 11-E6/E7 cells.

Conclusions: SR-T100 is effective for the treatment of human vulva condyloma, with few side effects. Compared with those with high-risk HPVs, cells with low-risk HPVs were more sensitive to SR-T100 treatment. Autophagy played a protective role in SR-T100-induced apoptosis in HPV-infected cells.

Trial registration: NCT01676792; Registered: August 29, 2012 (retrospectively registered).

Keywords: SR-T100, Condyloma, Hpv, Autophagy, Apoptosis

\footnotetext{
* Correspondence: d5580@mail.ncku.edu.tw

${ }^{1}$ Department of Obstetrics and Gynecology, National Cheng Kung University

Hospital, College of Medicine, National Cheng Kung University, 138 Sheng Li

Road, Tainan 704, Taiwan

Full list of author information is available at the end of the article
} 


\section{Background}

Genital warts or condyloma are the visible papules or papilliform tissues that are infected with human papillomaviruses (HPV), which consist of mostly HPV 6 and 11 $[1,2]$. It has been estimated that $75-80 \%$ of sexually active adults acquire a genital tract HPV infection before the age of 50 years. In addition to condyloma, some HPV genotypes have oncogenic potential, such as HPV16 and HPV-18, which have been recognized as the causative agents of cervical cancer. The transforming activity of oncogenic HPVs is provided by the E6 and E7 oncoproteins [3].

The primary strategy for treating warts is to remove the lesions. Chemical therapy aims to induce cell death. Podofilox arrests the tumor cell cycle, trichloroacetic acid (TCA) causes protein coagulation, and fluorouracil interferes with DNA synthesis. Immunologic therapy, such as imiquimod, induces cytokine production. Surgical methods via cryotherapy, laser ablation, or excisional therapy are reserved for condylomas that are not suitable for medical treatment or are refractory to the treatment modalities. Although each of these treatment modalities is efficacious in some patients, they are not completely without side effects and sequelae. Chemical and immunologic therapy may cause redness, swelling and pain, and surgical intervention may result in infection, bleeding, and/or poor cosmetic outcomes.

The anti-tumor effects of Solanum species have been previously reported, and several active ingredients, including solamargine, solasodine, and solasonine, suppress tumor growth in vitro and in vivo [4]. The mechanisms of the anti-tumor effect may be the increased expression of tumor necrosis factor receptor (TNFR) [5-7], or sensitization of the tumor cells to the cytotoxic agent $[8,9]$.

Recently, Solanum incanum extract (SR-T100), a water-soluble product that is primarily composed of the alkaloid solamargine, was shown to be an active treatment agent for skin squamous cell carcinoma (SCC) in vitro and in mice and in skin actinic keratosis patients [10]. In addition, SR-T100 may inhibit ovarian cancer formation by downregulating aldehyde dehydrogenase 1mediated stemness and chemoresistance [11].

The purpose of the present study was to evaluate the efficacy and safety of SR-T100 gel for the treatment of human condyloma and to study the possible underlying anti-condyloma mechanisms.

\section{Methods}

Safety and efficacy of SR-T100 gel on the vulvar condyloma acuminatum

The SR-T100 stock solution and SR-T100 gel were provided by G\&E Herbal Biotechnology Company (Tainan, Taiwan). SR-T100 is manufactured from the fruits of
Solanum incanum according to the patents (US patent 7,078,063, EU patent 1,058,334, and Japan patent $3,940,928)$. The chromatographic fingerprint of SR-T100 was shown in the Additional file 1: Figure S1. It revealed two major components, solamargine and solasonine as identified by comparisons with reference standards (APIN Chemicals, UK).

We conducted a pilot clinical study to evaluate the efficacy and safety of SR-T100 gel (2.3\% solamargine in Solanum incanum plant extract) in patients with external genital warts (EGW) or human vulvar condyloma acuminatum (ClinicalTrials.gov Identifier: NCT01676792). After obtaining approval from the Institutional Review Board (IRB No:HR-98-069) and informed consent from the patients, we enrolled patients at the Department of Obstetrics and Gynecology, National Cheng Kung University Hospital (Tainan, Taiwan). The detailed protocol and flow chart are shown in Additional file 1: Table S1.

Males or females older than 20 years of age who had at least one EGW with a lesion size greater than $5 \mathrm{~mm}$ in diameter were candidates for this study. The primary endpoint was to evaluate the treatment efficacy based on patients who received a 16-week treatment and presented evaluable measurement data by the end of the 20th week. The topical SR-T100 gel was self-applied to the lesion(s), including its peripheral normal skin approximately $1 \mathrm{~cm}$ around the lesion, in an amount of $0.02 \mathrm{~g} / \mathrm{cm}^{2}$ once daily with occlusive dressing. Patients who were unable to tolerate the occlusive dressing were instructed to apply the gel 3 times daily: in the morning, in the afternoon and before going to bed. It was recommended that the medication be re-applied after bathing or cleaning. Patient who had grossly suspicious or inflamed nodes on physical examination, had other organ site malignancy, currently under treatment and contraindicates this protocol therapy, use of any investigational drug in the 30 days before screening, pregnant or lactating women or women of childbearing potential using inadequate contraceptive methods, had immunodeficiency were excluded. The detailed protocol and flow chart are shown in Additional file 1: Table S1. Local skin reactions were assessed and graded as follows: $0=$ absent; 1 = mild (slight, barely perceptible); 2 = moderate (distinct presence); 3 = severe (marked, intense). The treatment response was evaluated by the tumor volume (length $\mathrm{x}$ width $\mathrm{x}$ height). A complete response (CR) was defined as $100 \%$ of targeted lesion cleared, a partial response (PR) was defined as $>75 \%$ of the targeted lesion cleared, progressive disease (PD) was defined as the development of new lesions or tumor growth, and stable disease (SD) was defined as lesions between PR and PD. The study nurse will guide the subjects to apply the gel before giving medications, and for each follow-up visit, subjects have to bring the medications back to check 
and weigh the used amount of medications to ensure patient's compliance. Patients who received a single local application of the SR-T100 gel were included in the analysis to determine their subsequent responses.

\section{Cell culture and reagents}

HeLa cells and human 293 embryonic kidney cells were purchased from the American Type Culture Collection (ATCC, Rockville, MD, USA). Cell lines were maintained in Dulbecco's modified Eagle's medium (Invitrogen, Carlsbad, CA, USA) supplemented with $10 \%$ fetal bovine serum (Sigma Chemical Co., St. Louis, MO, USA), $20 \mathrm{mM}$ HEPES, and $50 \mathrm{mg} / \mathrm{ml}$ gentamicin. For primary epithelial cells, normal uterine cervical cells were derived from patients who had undergone hysterectomy and were cultured in serum-free keratinocyte medium (Keratinocyte-SFM; Invitrogen) as previously described [12]. The 3methyladenine (3-MA), pepstatin A (PSTA), wortmannin (WT), trans-epoxysuccinyl-L-leucylamido-(4-guanidino)butane (E-64), benzyloxycarbonyl-valyl-alanyl-aspartic acid (O-methyl)-fluoromethylketone $\mathrm{z}$ (Z-VAD-FMK), monodansylcadaverine (MDC), and anti- $\beta$-actin antibody (clone AC-15; 1:25,000) were obtained from Sigma Chemical Co. DiOC6(3) was purchased from Molecular Probes. H2DCFDA was obtained from Thermo Fisher Scientific Inc. The Cell Titer Glo and Caspase-Glo ${ }^{\circ}$ 3, 8, 9 Assay kits were purchased from Promega (Madison, WI, USA). The primary antibodies were against LC3 (MBL, Nagoya, Japan), TNF-R1 (60192-1-Ig Proteintech, Chicago, USA), Fas (CD95 antibody N3C2, GeneTex, Inc.), and cleaved caspase-3 (\#9661) (Cell Signaling Technology, Inc.). Cisplatin (Fresenius Kabi Oncology Ltd., Haryana, India) was kindly provided by the Cancer Center at National Cheng Kung University Hospital, Tainan, Taiwan. The SR-T100 stock solution and SR-T100 gel (with 2.3\% of solamargine in Solanum incanum plant extract) were provided by G\&E Herbal Biotechnology Company (Tainan, Taiwan). SRT100 was manufactured from the fruits of S. incanum according to the patent (US patent 7,078,063, EU patent $1,058,334$, and Japan patent $3,940,928)$. In brief description, the active component of solamargine was extracted by acid-base precipitation and followed by the different ratios of ethanol/H2O extraction. The final extraction was then dried by lyophilization. Solamargine in SR-T100 extract was quantified by revers-phase high performance liquid chromatography, and diluted to a concentration of $10 \mathrm{mg} / \mathrm{ml}$ with pure water as a stock solution. SR-T100 contains solamargine and solasonine with the ratio approximately 65 to 35 , and less than $20 \%$ of hydrophilic minor components. Previous studies have demonstrated that solamargine, and to a lesser extent of solasonine, was the major active component of $S$. incanum against various tumor cells. [13]. Furthermore, other components within SR-T100 did not show cytotoxicity to tumor cells [10].
Thus, solamargine serves as standard in the present study for quality control. Since the SR-T100 gel is conducting clinical trials under US and Taiwan FDA approvals, the analytical method for quantifying SR-T100 gel is validated according to the standard pharmaceutical procedures.

\section{Condyloma sample collection, DNA extraction and HPV genotyping}

Condyloma samples were obtained by evenly swabbing the lesions using a cytobrush. The brush was then placed in a cone-shaped tube with transport medium for DNA extraction and genotyping. The DNA samples were extracted using the QIAamp DNA Blood Mini Kit (QIAGEN, Cat. No. 51104). We used the HPV Blot chip (Easychip HPV Blot, King Car Co., I-Lan, Taiwan) as previously described [14] to detect and genotype the HPV DNA in the lesions. The assay is based on the detection of a fragment in the L1 open reading frame of HPV and can detect 39 types of HPV DNA in a single reaction. HPV was classified into high-risk or low-risk types according to the report by Muñoz et al. [15]. In this study, we classified probable high-risk types (i.e., 26, 53, and 66) as high-risk HPV types.

\section{Construction of HPV6b, 11, 16, 18, -E6 and -E7 lenti- plasmids, lentiviral particles and HPV-associated primary epithelial cells}

The lentiviral vectors, pWPXL/HPV-E6 and -E7 were constructed by replacing the GFP coding region in pWPXL/GFP, which was provided by Professor Wu CL in the Department of Biochemistry and Molecular Biology, National Cheng Kung University (Tainan, Taiwan), with the E6 or E7 DNA fragments of HPV 6b, HPV11, HPV16 and HPV18 at MluI/EcoRI sites. The pGEMHPV 6b, -HPV11, -HPV16, and -HPV18 plasmids were kindly provided by Dr. Huang CC in the Department of Pathology, Kaohsiung Chang Gung Memorial Hospital (Kaohsiung, Taiwan). The different types of HPV-E6 and -E7 fragments were obtained by polymerase chain reaction (PCR) amplification from different type of HPV plasmids, in which a BglII site and an EcoRI site were introduced in the $5^{\prime}$-end and the $3^{\prime}$-end, respectively. The detailed sequences of the PCR primers are listed in Additional file 1: Table S2. The PCR products were digested with $B g l \mathrm{II} / E c o$ RI, cloned into pcDNA3-HAHIF1 at by BamHI/EcoRI sites, and further sub-cloned into pWPX at $M l u \mathrm{I} / E c o$ RI sites. Various recombinant lentiviruses were produced by transiently transfecting $293 \mathrm{~T}$ cells with pWPXL/GFP or pWPXL/Null; pWPXL/ HPV6b-E6 or -E7; pWPXL/HPV11-E6 or -E7; pWPXL/ HPV16-E6 or -E7; or pWPXL/HPV18-E6 or -E7, with the packaging plasmid psPAX2 and the VSV expression plasmid pMD2.G using the calcium phosphate 
precipitation method. After $48 \mathrm{~h}$, the lentiviral particles were collected from the supernatant.

Ten lentiviral particles were produced, including Lenti-GFP, Lenti-Null (no transgenes), Lenti-HPV6b-E6, Lenti-HPV6b-E7, Lenti-HPV11-E6, Lenti-HPV11-E7, Lenti-HPV16-E6, Lenti-HPV16-E7, Lenti-HPV18-E6 and Lenti-HPV18-E7, which were titrated using a previously described method $[16,17]$. Normal cervical epithelial cells were simultaneously infected with a MOI of 5 Lenti-HPV6b-E6 and Lenti-HPV6b-E7 for $24 \mathrm{~h}$ to produce HPV6b-E6/E7-expressing primary epithelial cells (HPV6b cells). The same procedures were used to produce the Null (Null cells), HPV11-E6/E7 (HPV11 cells), HPV16-E6/E7 (HPV16 cells) and HPV18-E6/E7 (HPV18 cells) primary epithelial cells.

\section{RNA extraction and reverse transcriptase-polymerase chain reaction (RT-PCR)}

RT-PCR was used to confirm the expression of the different types of HPV E6 and E7 genes in the primary epithelial cells. Total RNA was extracted from the different types of HPV E6/E7 primary epithelial cells using the RNeasy Mini kit (Qiagen, Valencia, CA). Total RNA (2 $\mu \mathrm{g})$ was reverse transcribed to cDNA using reverse transcriptase (Promega Corporation, Madison, WI, USA.) Briefly, the RNA samples were heated with RNase-free $\mathrm{H}_{2} \mathrm{O}$ and oligo-dT(15) primers (Promega Corporation, Madison, WI, USA) for $5 \mathrm{~min}$ at $70{ }^{\circ} \mathrm{C}$ and immediately cooled on ice. Subsequently, a deoxyribonucleoside triphosphate (dNTP), $5 \times$ first strand buffer, RNase inhibitor, and MMLV (Moloney murine leukemia virus) reverse transcriptase were gently mixed and then incubated at $42{ }^{\circ} \mathrm{C}$ for $60 \mathrm{~min}$. The HPVE6/E7 primers are described above. RTPCR was performed on a thermal cycler (Applied Biosystems, Foster City, CA, USA) using GoTaq Green Master Mix (Promega Corporation, Madison, WI, USA.). The PCR products were resolved on $2 \%$ agarose gels. GADPH was used as an internal control.

\section{Cell viability assay}

The CellTiter-Glo ${ }^{\circ}$ Cell Viability Assay (Promega, Madison, WI, USA) according to the manufacture's protocol was used to determine the cell viability after SR-T100 treatment. Briefly, $1 \times 10^{5}$ normal cervical epithelial cells/well were grown in 96-well plates overnight at $37^{\circ} \mathrm{C}$ and then simultaneously infected with Lenti-HPV6b-E6 or -E7 (to produce HPV6b cells), Lenti-HPV11-E6 or -E7 (to produce HPV11 cells), Lenti-HPV16-E6 or -E7 (to produce HPV16 cells), or Lenti-HPV18-E6 or -E7 (to produce HPV18 cells) for $24 \mathrm{~h}$. The cells were then further treated with the indicated concentrations of SR-T100 $(\mu \mathrm{g} / \mathrm{ml})$ for $24 \mathrm{~h}$. Dose-inhibition curves and the $\mathrm{IC}_{50}$ of SR-T100 were calculated for the different types of HPV-E6/E7-infected primary epithelial cells using commercial software (SigmaPlot V10.0, Systat Software, Inc., Richmond, CA, USA).

\section{Cell death measurements}

The distribution of apoptotic cells was determined using the FITC Annexin V Apoptosis Detection Kit I (BD Pharmingen $\left.^{\mathrm{Tm}}\right)$ as previously reported [18]. Briefly, different types of HPV-E6/E7 primary epithelial cells were treated with SR-T100 for $12 \mathrm{~h}$. In another set of experiment, SR-T100-treated cells were treated concomitantly with the autophagy inhibitors WT $(200 \mathrm{nM})$ for $3 \mathrm{~h}$ or 3-MA ( $2.5 \mathrm{mM})$ for $30 \mathrm{~min}$. All cells were collected and resuspended in 1X Binding Buffer at a concentration of $1 \times 10^{6}$ cells $/ \mathrm{ml}$. The Annexin $\mathrm{V}$ and/or propidium iodide (PI) reagents were added to $100 \mu \mathrm{L}$ of cells in a microtube and incubated in the dark for $20 \mathrm{~min}$ at room temperature. The cells were then analyzed using $\mathrm{BD}$ FACSDiva software and a FACSCanto II flow cytometer (BD Biosciences).

\section{Detection of caspase activity}

Caspase-3, -8 and -9 activities were assayed using a luminescent assay kit (Promega, Madison, WI, USA) according to the manufacturer's instructions. Briefly, the different types of HPV-E6/E7 primary epithelial cells were treated with SR-T100 $(5 \mu \mathrm{g} / \mathrm{ml})$ for $12 \mathrm{~h}$. The samples were mixed with substrates and incubated in a $25^{\circ} \mathrm{C}$ incubator for $2 \mathrm{~h}$ in the dark. The plate was read on a Luminoskan Ascent microplate luminometer (Thermo). The changes in caspase activity were calculated as the percent change in the absorbance (luminescence) of the cells treated with or without SR-T100.

\section{Measurement of mitochondrial membrane potential (MMP)}

The MMP was measured by assessing DiOC6(3) uptake to detect the mitochondrial apoptosis pathway, as previously described [19]. Briefly, different types of HPV-E6/ E7 primary epithelial cells were treated with SR-T100 for $12 \mathrm{~h}$, followed by staining with $40 \mathrm{nM}$ DiOC6(3) for $30 \mathrm{~min}$ at $37^{\circ} \mathrm{C}$ in the dark. The MMP was determined by measuring the retention of DiOC6(3) with a FACSCanto II flow cytometer (BD Biosciences). The data were sorted by analyzing 20,000 events using BD FACSDiva software. The fraction of cells (M1) with low MMP levels was identified by flow cytometry.

\section{Visualization and quantitative analysis of intracellular autophagic vacuoles}

MDC staining and GFP-LC3 aggregation were used to quantify the autophagic vacuoles as previously described [18]. For the MDC staining experiment, different types of HPV-E6/E7 primary epithelial cells were grown on coverslips, treated with SR-T100 for $12 \mathrm{~h}$, incubated with 
$0.05 \mathrm{mM}$ MDC (Sigma) for $10 \mathrm{~min}$ at $37^{\circ} \mathrm{C}$, washed four times with PBS, and immediately analyzed by fluorescence microscopy (Olympus BX51, Tokyo, Japan). Images of the MDC-labeled vacuoles were obtained with a CCD camera. For the GFP-LC3 aggregation assay, normal cervical epithelial cells were first transfected with pGFP-LC3 using electroporation with the Neon ${ }^{\circ}$ Transfection System (Thermo), then infected with different types of Lenti-HPV-E6 and -E7 viruses, and treated with SR-T100 for $12 \mathrm{~h}$. The cells were subsequently analyzed by fluorescence microscopy. The number of MDCpositive or GFP-LC3-positive dots was then counted. At least 50 cells were counted in each microscopic field at $200 \times$ magnification. A total of five different fields were investigated.

\section{Transmission electron microscopy (TEM)}

We used transmission electron microscopy to observe the numerous autophagic vacuoles with a doublemembrane structure as previously described [18]. Different types of HPV-E6/E7 primary epithelial cells were treated with or without SR-T100 for $12 \mathrm{~h}$, washed with $0.1 \mathrm{M}$ phosphate buffer $(\mathrm{pH}$ 7.2), and prefixed in the same buffer containing $2 \%$ formaldehyde and $2.5 \%$ glutaraldehyde in situ for $24 \mathrm{~h}$ at $4{ }^{\circ} \mathrm{C}$. The samples were postfixed in $1 \%$ osmium tetroxide $(\mathrm{OsO} 4)$ for $1.5 \mathrm{~h}$ at $4{ }^{\circ} \mathrm{C}$, dehydrated in increasing concentrations of ethanol and pure propylene oxide (PO), embedded in Embed-812 resin at room temperature, and polymerized in a $60{ }^{\circ} \mathrm{C}$ oven for $20 \mathrm{~h}$. The samples $(90 \mathrm{~nm})$ were cut from the blocks and then contrasted with lead citrate and uranyl acetate. The samples were viewed with an H-7650 transmission microscope (HITACHI, Japan).

\section{Western blotting}

Western blotting was used to determine LC3-I/II, TNFR1, and FAS expression in the different types of HPVE6/E7 primary epithelial cells treated with or without SR-T100. LC3 was detected and quantified by western blotting to monitor autophagy activity. In the LC3-I/II expression experiment, the protease inhibitors PSTA $(10 \mu \mathrm{g} / \mathrm{ml})$ and E-64 $(10 \mu \mathrm{g} / \mathrm{ml})$ were added to the culture medium before SR-T100 treatment to prevent degradation of the LC3 protein. Equal amounts of protein from the lysates of cells subjected to various treatments were resolved by SDS-PAGE and transferred to Hybond $^{\mathrm{m}}-\mathrm{P}$ PVDF transfer membranes (Amersham Biosciences). After incubation with the indicated primary antibody, the membrane was incubated with an HRPconjugated secondary antibody. Antibody binding was detected by enhanced chemiluminescence followed by exposure to X-ray films or the BioSpectrum ${ }^{\circ}$ Imaging System (UVP). The LC3-II levels were further normalized to the levels of the "housekeeping" protein actin to monitor autophagy, as suggested in published guidelines [20]. The LC3-II/actin ratio was calculated based on the densitometry analysis of both bands.

\section{Statistics}

The experimental results were expressed as mean \pm S.D. For the SR-T100 inducing growth inhibition and apoptotic cell death in primary epithelial cells expressing different types of HPV-E6/E7 assays, the data were analyzed using a two-way analysis of variance (ANOVA). Student's t-test or one-way ANOVA was used to assess two or multiple groups with Newman-Keuls post hoc test. The association between HPV types and SR-T100 response was analyzed using Fisher's exact test. A Pvalue $<0.05$ was considered statistically significant. All analyses were performed using the SPSS statistical software package (SPSS Statistics version 16, SPSS Corporation, Chicago, IL, USA).

\section{Results}

The SR-T100 gel is effective and safe for the treatment of vulvar condyloma

A total of 26 patients were enrolled into this study. Among them, 16 patients completed the treatment, 6 patients withdrew consent because of concerns regarding the treatment schedule, 3 patients decided to receive other treatments due to a poor response to SR-T100, and one patient was lost to follow-up after the 6th visit. All the patients received at least one local application of the SR-T100 gel. The clinical information, treatment response and HPV typing of the patients are shown in Table 1. Sixteen cases had complete response $(100 \%$ clearance of lesions); the rate of complete clearance for SR-T100 was $61.5 \%(16 / 26)$ in this study. Three cases showed a partial response ( $>75 \%$ lesion volume reduction). Therefore, the response rate of SR-T100 in this study was $73 \%(19 / 26)$.

For the HPV types, 13 cases were only infected with low-risk HPV types, and 13 cases were infected with a mixture of low and high-risk HPV types. HPV 6 was the most common HPV type and was observed in approximately $61.5 \%(16 / 26)$ of patients. HPV11 was the second most common HPV type and was observed in approximately $30.7 \%(8 / 26)$ of the patients in this study.

Regarding the side effects of the SR-T100 gel used in this study, 2 patients had moderate stinging/burning sensations in the skin, and 3 patients had grade 2 to 3 itching. One patient experienced severe stinging/burning sensations that caused her to withdraw from the trial.

\section{Cells expressing high-risk type HPV E6/E7 are resistant to SR-T100-induced apoptosis}

Based on the HPV typing and treatment response analyses, we found that patients infected with high-risk 
Table 1 Summary of patients with condyloma treated with SR-T100 gel

\begin{tabular}{|c|c|c|c|c|c|c|}
\hline Patient & Age (y/o) & $\begin{array}{l}\text { Maximum } \\
\text { diameter (mm) }\end{array}$ & $\begin{array}{l}\text { Lesion } \\
\text { volume }\left(\mathrm{mm}^{3}\right)\end{array}$ & $\begin{array}{l}\text { Treatment } \\
\text { duration(wk) }\end{array}$ & Outcome & HPV types \\
\hline 1 & 55 & 5.0 & 125 & 16 & $C R$ & 6 \\
\hline 2 & 30 & 7.4 & 131 & 16 & $C R$ & $6 / 54$ \\
\hline 3 & 32 & 5.0 & 50 & 16 & $C R$ & $31 / 44 / 53$ \\
\hline 4 & 29 & 16.9 & 1233 & 16 & $C R$ & $6 / 54$ \\
\hline $5^{a}$ & 24 & 7.8 & 35 & 4 & SD & $11 / 39 / 62$ \\
\hline 6 & 36 & 6.3 & 66 & 16 & $C R$ & $42 / 67$ \\
\hline $7^{b}$ & 52 & 15.3 & 123 & 12 & SD & 66 \\
\hline 8 & 70 & 29.2 & 2397 & 16 & $P R$ & $11 / 62$ \\
\hline 9 & 63 & 9.3 & 256 & 16 & $C R$ & 11 \\
\hline 10 & 40 & 7.0 & 72 & 16 & $C R$ & $6 / 58$ \\
\hline $11^{\mathrm{a}}$ & 36 & 6.0 & 52 & 4 & $P R$ & $6 / 81$ \\
\hline 12 & 26 & 12.2 & 117 & 16 & $C R$ & $6 / 70$ \\
\hline 13 & 31 & 5.1 & 98 & 16 & $C R$ & 6 \\
\hline $14^{\mathrm{a}}$ & 34 & 17.3 & 416 & 2 & SD & $6 / 16$ \\
\hline 15 & 25 & 6.3 & 74 & 16 & $C R$ & 6 \\
\hline 16 & 57 & 5.5 & 21 & 16 & $C R$ & $6 / 35$ \\
\hline $17^{b}$ & 45 & 5.0 & 38 & 8 & PD & 6/67/18 \\
\hline 18 & 71 & 12.8 & 87 & 16 & $C R$ & $11 / 84 / 82$ \\
\hline $19^{\mathrm{a}}$ & 30 & 6.5 & 95 & 16 & $P R$ & $6 / 51 / 58$ \\
\hline $20^{\mathrm{a}}$ & 26 & 5.3 & 2 & 4 & SD & $6 / 54$ \\
\hline $21^{c}$ & 38 & 7.6 & 4 & 12 & $C R$ & 6 \\
\hline 22 & 22 & 11.3 & 20 & 12 & $C R$ & $11 / 16 / 84$ \\
\hline $23^{\mathrm{a}}$ & 38 & 13.0 & 162 & 2 & SD & $11 / 31 / 58 / 67$ \\
\hline $24^{b}$ & 30 & 19.0 & 161 & 8 & PD & 6/11/16 \\
\hline 25 & 39 & 20.5 & 214 & 16 & $C R$ & 6 \\
\hline 26 & 28 & 5.5 & 42 & 16 & $C R$ & $11 / 58$ \\
\hline
\end{tabular}

$C R$ complete response (100\% cleared), $P R$ partial response (> 75\% cleared), SD stable disease, $P D$ progressive disease, $w k$. week

a patient with withdrew consent

b patient who decide to receive other treatment

cpatient lost to follow-up

HPV types were less responsive to SR-T100 treatment ( $p$ $=0.027$, Fisher's exact test, Table 2). We generated 10 recombinant lentiviruses and produced $4 \mathrm{HPV}$-containing primary cells that expressed low or high-risk HPV E6/E7 types, as described in Materials and Methods section, to investigate the effects of low or high-risk types of HPV infection on SR-T100 treatment. The cells were treated with the indicated concentrations of SR-T100 for $24 \mathrm{~h}$, and the cell viabilities were calculated and the low or high-risk

Table 2 HPV types and SR-T100 response in condyloma $(N=26)$

\begin{tabular}{lll}
\hline HPV types & Response $(N=19)$ & Non-response $(N=7)$ \\
\hline $\begin{array}{l}\text { Mixed low and } \\
\text { high risk HPV types }\end{array}$ & 7 & 6 \\
6,11 or low risk & 12 & 1 \\
HPV types only & & \\
$P=0.027$, Fisher's exact test &
\end{tabular}

type HPV E6/E7 expression in cervical epithelial cells was confirmed by RT-PCR as shown in Fig. 1a.

These results suggested that SR-T100 induced cell death and inhibited cell proliferation in a dosedependent manner. The $\mathrm{IC}_{50}$ values of SR-T100 in the HPV6b, HPV11, HPV16, and HPV18-E6/E7 cells were $5.1,5.2,6.7$, and $6.5 \mu \mathrm{g} / \mathrm{ml}$, respectively. Cells expressing high-risk types of HPV-E6/E7 were more resistant to SR-T100 treatment. Apoptotic cell death was evaluated using Annexin V/PI double staining to further determine whether SR-T100-induced cell death was mediated by apoptosis. The flow cytometry analysis showed that SRT100 induced apoptosis in HPV-expressing primary cells (Fig. 1b). These data suggested that apoptotic cell death events contributed to the growth inhibitory effect of SRT100. When the cells were treated with SR-T100 $(5 \mu \mathrm{g} /$ $\mathrm{ml}$ ) for $12 \mathrm{~h}$, the percentages of apoptotic HPV 6b-E6/ 


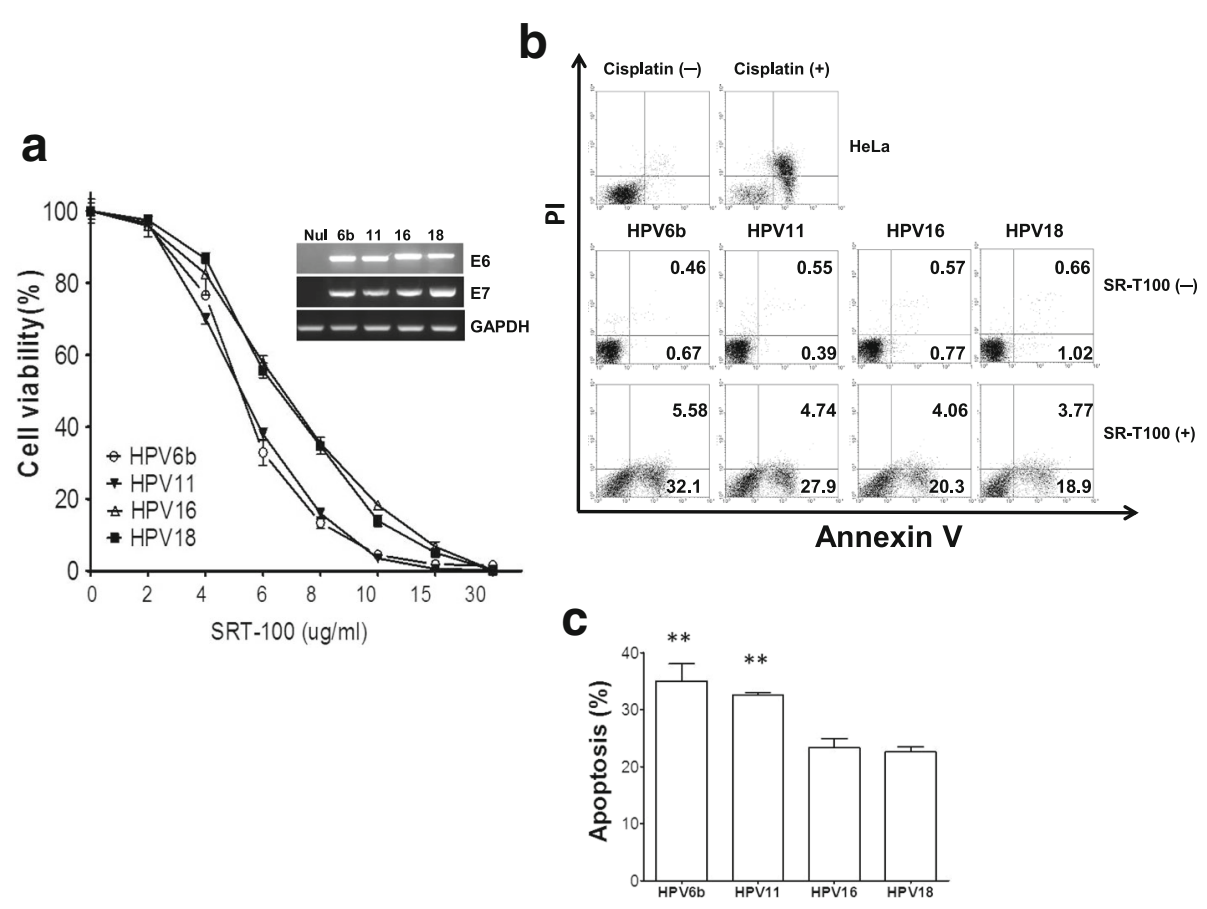

Fig. 1 SR-T100 induced different levels of growth inhibition and apoptotic cell death in primary epithelial cells expressing different types of HPVE6/E7. a After infection with different types Lenti-HPVE6/E7 viruses, the primary epithelial cells were treated with the indicated concentrations of SR-T100 for $24 \mathrm{~h}$. Cell viability was determined using the CellTiter-Glo luminescent cell viability assay. Each value represents the mean \pm SD of three determinations. HPV-E6/E7 expression in normal cervical epithelial cells was confirmed by RT-PCR as shown in the inset panel. $\mathbf{b}$ HeLa cells were treated with cisplatin $(150 \mu \mathrm{M})$ for $24 \mathrm{~h}$ (upper panel) and used as a positive control. After infection with different types of Lenti-HPV-E6/E7 viruses, the primary epithelial cells were treated with SR-T100 $(5 \mathrm{\mu g} / \mathrm{ml})$ for $12 \mathrm{~h}$ and double labeled with Annexin V-FITC and propidium iodide (PI). In the cytograms from three experiments, the apoptotic cells (Annexin V-positive) are distributed in the lower and upper right panels (the values are reported as percentages). c Histograms showing the number of apoptotic primary epithelial cells expressing different types of HPV-E6/ E7. The results shown are the mean values of three independent experiments; bars, \pm SD. ${ }^{* *} p<0.01$, HPV6 or HPV11 versus HPV16 or HPV18

E7 and HPV11-E6/E7 cells were approximately 37\% and $31 \%$, respectively, which was significantly increased compared with HPV16-E6/E7 cells (approximately 24\%) or HPV18-E6/E7 cells (approximately 21\%) (Fig. 1c).

\section{SR-T100 induces mitochondria-dependent apoptosis in HPV-expressing cells, particularly in cells expressing the low-risk HPV-E6/E7 type}

Previously, SR-T100 was shown to induce skin cancer apoptosis through both tumor necrosis factor receptor (TNFR) signaling and mitochondrial pathways [10]. Mitochondrial function is critical for cell viability. Disruption of the mitochondrial membrane potential may irreversibly induce cell apoptosis. In this study, we did not observe increased TNF-R1 and Fas expression (Fig. 2a) or caspase- 8 activation (Fig. 2d), but we did observe a loss of MMP (Fig. 2b-c) and caspase-9 and -3 activation (Fig. 2d) in HPV-infected cells after treatment with SR-T100. In addition, the percent changes in MMP and caspase- 9 and -3 activities were significantly increased in HPV6b-E6/E7- and HPV11-E6/E7-expressing cells compared with HPV16-E6/E7- and HPV18-E6/E7expressing cells. Our data suggested that SR-T100 induced apoptosis through the mitochondrial pathway in HPV-infected primary epithelial cells and was more effective in cells infected with low-risk HPV types.

\section{SR-T100 induced a greater autophagic response in HPV16-E6/E7 and HPV18-E6/E7 cells than in HPV6b-E6/E7 and HPV11-E6/E7 cells}

Autophagy, a highly conserved catabolic process for the degradation of proteins and organelles, has been shown to act as a pro-survival or pro-death mechanism under different physiological and pathological conditions [21]. HPV-infected cells were treated with SR-T100 $(5 \mu \mathrm{g} / \mathrm{ml})$ for $12 \mathrm{~h}$ and assayed for LC3 expression, GPF-LC3 aggregation, and MDC staining using fluorescence microscopy. In addition, autophagy was assessed by TEM to evaluate its involvement in the effects of SR-T100 treatment (Fig. 3). Following the induction of autophagy, LC3 is modified to a membrane-bound form, LC3-II (a LC3-phospholipid conjugate), which translocates to and aggregates on the membrane of autophagic vacuoles [22]. LC3 aggregation and LC3-II conversion are widely used as indicators of autophagy [20]. As shown in Fig. 3a, the fold changes in the LC3-II/actin ratio between cells 


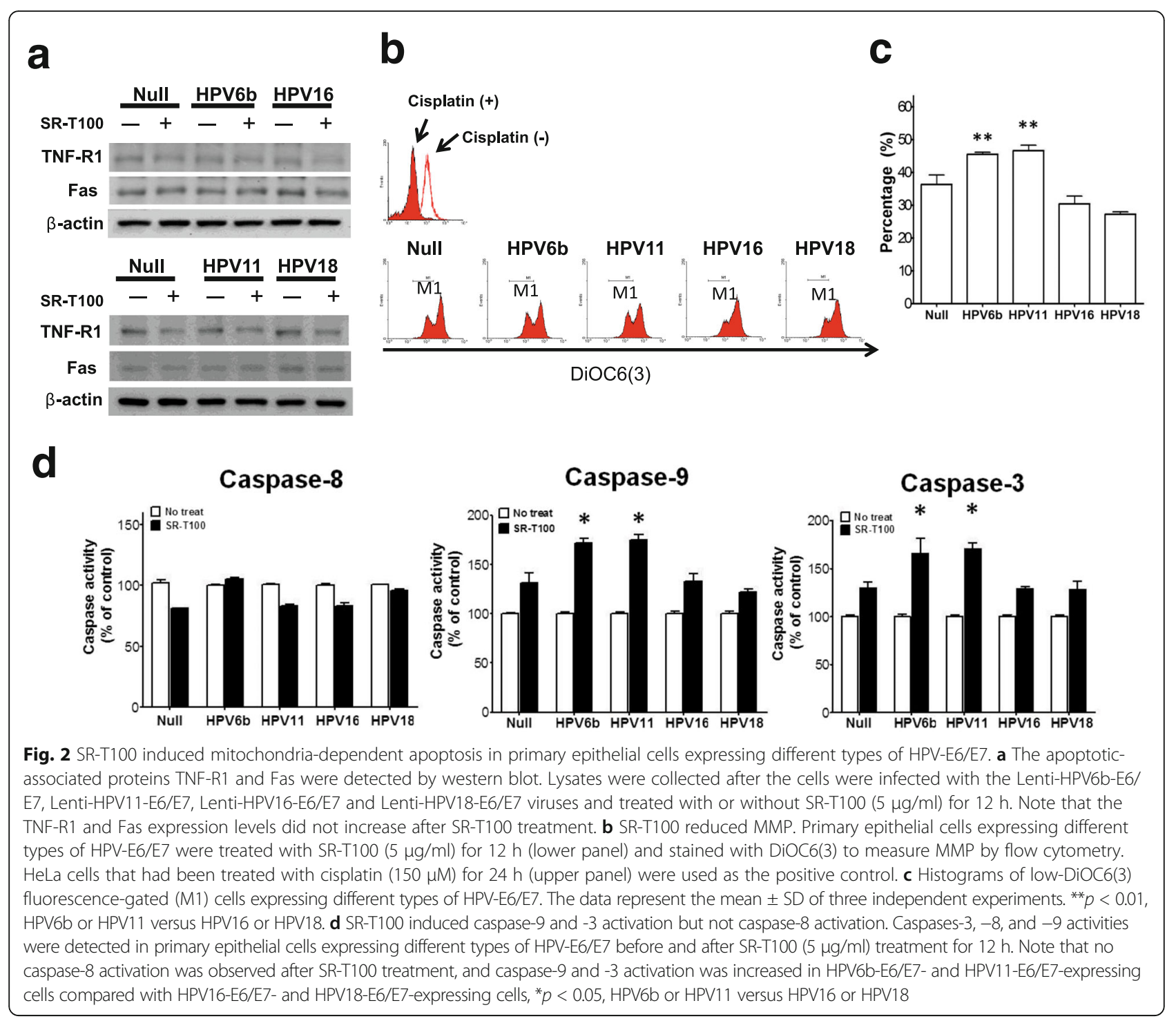

treated with SR-T100 and cells without SR-T100 treatment were 1.2, 1.3, 1.3, 1.7, and 1.5 in HPV Null, HPV6b-, HPV11-, HPV16-, and HPV18-E6/E7 cells, respectively. As shown in Fig. 3b, the number of GFPLC3-positive dots was significantly increased among cells expressing HPV16-E6/E7 and HPV18-E6/E7 compared with cells expressing HPV6b-E6/E7 and HPV11E6/E7. The MDC fluorescent dye has been suggested to be a specific marker for autophagic vacuoles. In Fig. 3c, cells expressing HPV16-E6/E7 and HPV18-E6/E7 also displayed more punctate MDC fluorescence than cells expressing HPV6b-E6/E7 and HPV11-E6/E7 after SRT100 treatment. We also observed autophagic vacuoles with a double-membrane structure after SR-T100 treatment using transmission electron microscopy to further verify that SR-T100 could induce autophagy in HPVexpressing cells, particularly HPV16-E6/E7-expressing cells (Fig. 3d).
Autophagy inhibition enhances SR-T100-induced apoptosis in HPV 16-E6/E7 and HPV18-E6/E7 cells, whereas apoptosis inhibition enhances SR-T100-induced autophagy in HPV 6b-E6/E7 and HPV11-E6/E7 cells

We first used the autophagy inhibitors WT and 3-MA to block autophagy and study its relationship with apoptosis in HPV-expressing SR-T100-treated cells, as previously reported [18]. LC3-II expression decreased when cells were treated with WT or 3-MA (Fig. 4a). The LC3II/actin ratio in SR-T100-treated HPV16-E6/E7 cells was clearly reduced from 1.4 to 1.1 or 1.0 following treatment with WT or 3-MA, and from 1.3 to 1.0 or 0.9 in SR-T100-treated HPV18-E6/E7 cells. In addition, as shown in Fig. 4b, inhibition of autophagy by WT or 3MA significantly increased apoptosis in high-risk type HPV-infected cells following treatment with SR-T100. The WT or 3-MA treatment significantly increased the rate of apoptosis from $22.89 \%$ to $29.96 \%$ or $34.80 \%$ in 


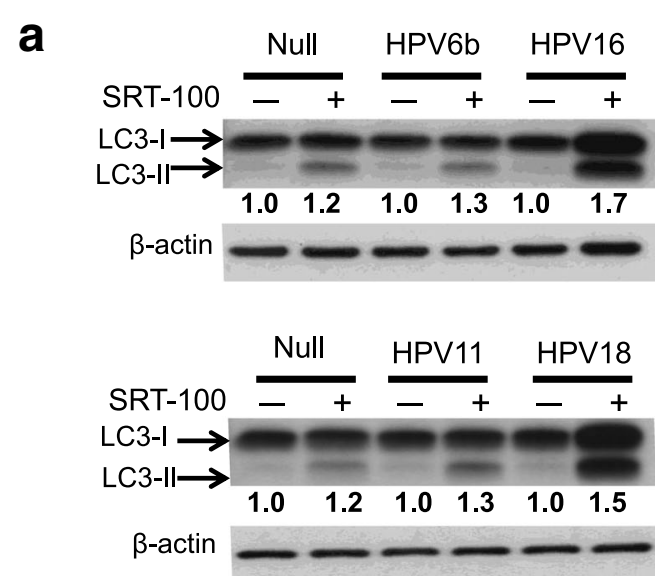

b

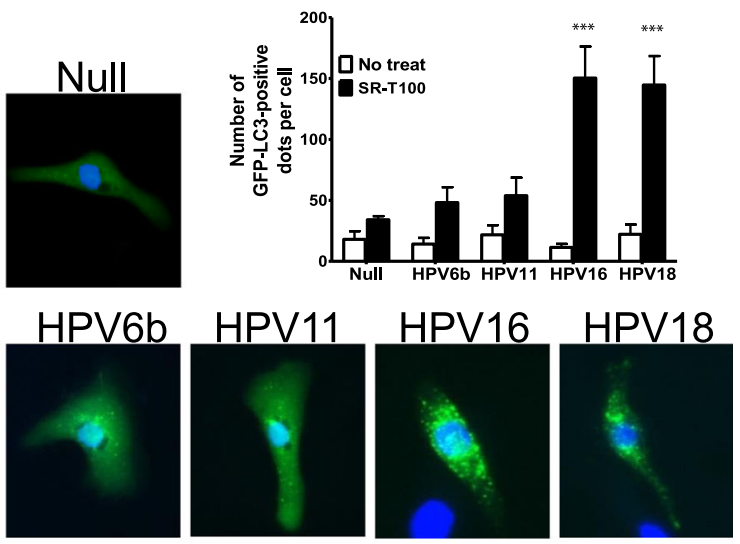

d

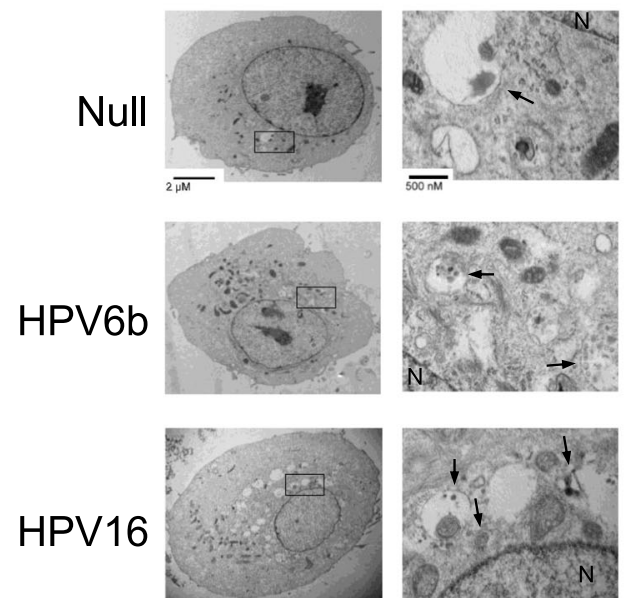

Fig. 3 SR-T100 induced a greater autophagic response in primary epithelial cells expressing HPV16-E6/E7 and HPV18-E6/E7. a Immunoblotting for LC3-I and LC3-II using lysates from primary epithelial cells expressing different types of HPV-E6/E7 treated with or without SR-T100 (5 $\mu$ g/ml) for $12 \mathrm{~h}$. Similar results were observed in at least three independent experiments. The number indicates the fold change in the LC3-II/actin ratios in cells treated with SR-T100 versus those without SR-T100 treatment. b Activation of autophagy was observed in primary epithelial cells expressing different types of HPV-E6/E7 that were treated with SR-T100 $(5 \mu \mathrm{g} / \mathrm{ml})$ for $12 \mathrm{~h}$ using GFP-LC3 accumulation. The cells were first transfected with LC3-GFP, infected with different types of Lenti-HPV-E6/E7 viruses, and then treated with SR-T100 for 12 h. GFP-LC3 accumulation was analyzed under a fluorescence microscope (400x), followed by quantitation of the number of green spots per cell showing LC3 accumulation in cytoplasmic vacuoles (inset panel). ( ${ }^{* *} p<0.001$ : HPV16 or 18 versus HPV6b or 11 ). Representative images of cells that were treated with SR-T100 for $12 \mathrm{~h}$ are shown. c Visualization of the activation of autophagy in primary epithelial cells expressing different types of HPV-E6/E7 that were treated with SR-T100 (5 $\mathrm{mg} / \mathrm{ml})$ for $12 \mathrm{~h}$ using MDC staining. The formation of autophagic vacuoles was suggested by the interspersed MDC labeling in the cytoplasm observed under a fluorescence microscope (400X). Representative images of cells that were treated with SR-T100 for $12 \mathrm{~h}$ are shown. Quantitative results of MDC staining from three independent experiments (** $p<0.001$ : HPV16 or HPV18 versus HPV6b or HPV11). d Ultrastructural images of the SR-T100-treated cells were obtained using an electron microscope. Primary epithelial cells were infected with or without HPV6b-E6/E7 or HPV16-E6/E7 lentiviruses for $24 \mathrm{~h}$ and treated with SR-T100 (5 $\mu \mathrm{g} / \mathrm{ml})$ for $12 \mathrm{~h}$. The right panel (25,000x) shows the magnified image of the area indicated by the box in the left panel (8,000x). Autophagic vacuoles are denoted by arrows. Note the increased number of autophagic vacuoles in cells expressing HPV16-E6/E7. N, nucleus

SR-T100-treated HPV16-E6/E7 cells, and from 20.80\% to $27.34 \%$ or $34.25 \%$ in SR-T100-treated HPV18-E6/E7 cells. Furthermore, to understand the role of apoptosis in SR-T100-induced autophagy, we used the pan-caspase inhibitor Z-VAD-FMK to repress apoptosis. Caspase-3 cleavage was blocked and caspase- 3 activity was reduced in HeLa cells treated with cisplatin in the presence of ZVAD-FMK $(50 \mu \mathrm{M})$ (Additional file 1: Figure S2a-b). In
HPV-infected cells, caspase- 3 activity was reduced and cell viability was recovered in response to treatment with SR-T100 in the presence of Z-VAD-FMK $(50 \mu \mathrm{M})$, particularly in HPV6b-E6/E7 and HPV11-E6/E7 cells (Additional file 1: Figure S2c-d).

As shown in Fig. 4c, LC3-II expression increased following treatment with SR-T100 in the presence of ZVAD-FMK, particularly in cells infected with the low- 

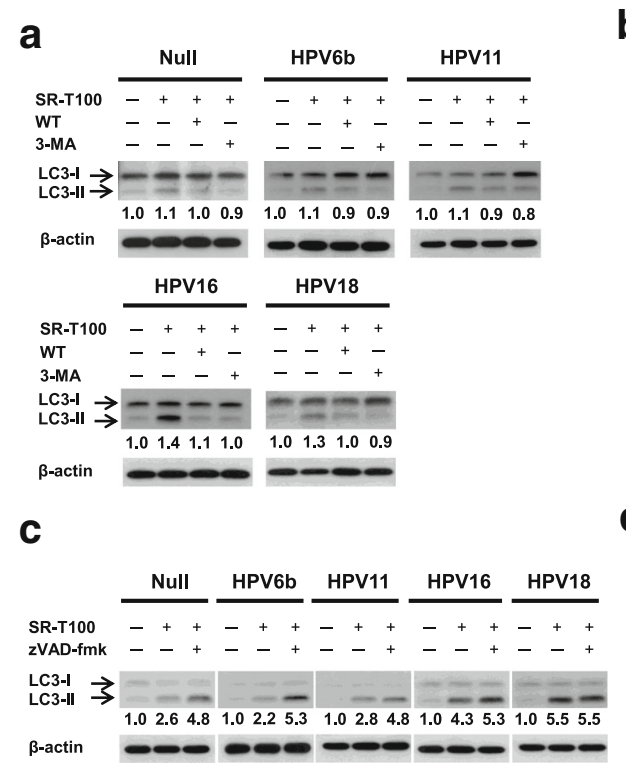

b
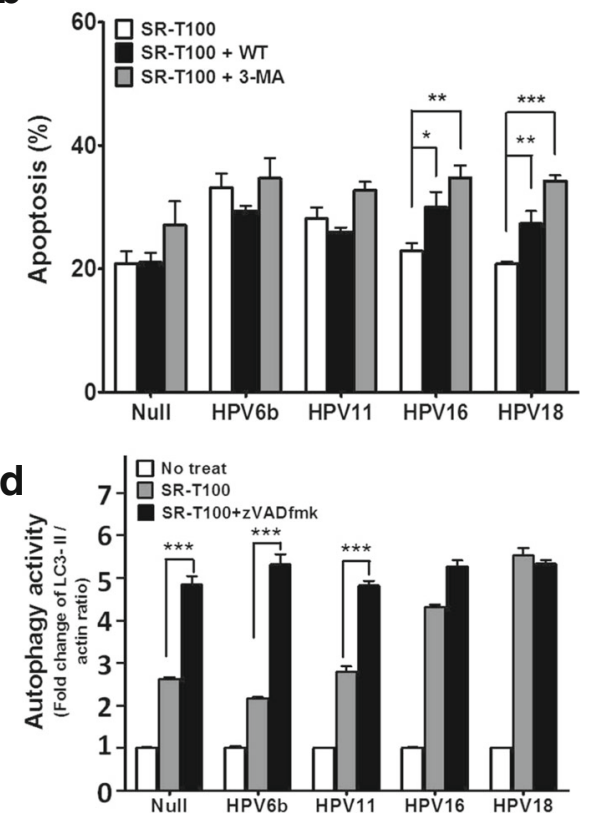

Fig. 4 Inhibition of autophagy resulted in increased apoptotic cell death in SR-T100-treated primary epithelial cells expressing HPV 16-E6/E7 and HPV18-E6/E7. a Wortmannin (WT) and 3-MA inhibited early autophagy activities in SR-T100-treated primary epithelial cells expressing HPV-E6/E7. Immunoblotting for LC3I/I using lysates from primary epithelial cells expressing different types of HPV-E6/E7 that were pretreated with the autophagy inhibitors wortmannin (WT) $(200 \mathrm{nM})$ or 3-MA (2.5 mM) and then treated with or without SR-T100 $(5 \mu \mathrm{g} / \mathrm{ml})$ for $12 \mathrm{~h}$. The blots were stripped and reprobed with an anti- $\beta$-actin antibody to ensure equal protein loading. Similar results were observed in three independent experiments. The number indicates the fold change in the LC3-II/actin ratios in cells treated with SR-T100 versus those without SR-T100 treatment. $\mathbf{b}$ Histograms show the number of apoptotic SR-T100-treated $(5 \mathrm{\mu g} / \mathrm{ml}$ ) cells (Annexin V-positive) that were incubated in the presence or absence of WT and 3-MA for $12 \mathrm{~h}$. The cells were incubated with SR-T100 $(5 \mu \mathrm{g} / \mathrm{ml})$ in the presence or absence of the autophagy inhibitors WT or 3-MA for $12 \mathrm{~h}$. At the end of the treatments, the cells were double labeled with Annexin V-FITC and propidium iodide (PI) and analyzed by flow cytometry to evaluate cell viability. WT and 3-MA significantly increased apoptotic cell death in primary epithelial cells expressing HPV16-E6/E7 and HPV18-E6/E7. The data represent the mean \pm SD from three independent experiments. ${ }^{*} P<0.05$; ${ }^{*} P<0.01$. c The LC3-I and LC3-II proteins were detected by western blotting. The primary epithelial cells were infected with different types Lenti-HPV-E6/E7 virus for $24 \mathrm{~h}$. After $24 \mathrm{~h}$, the infected cells were incubated in culture medium in the presence or absence of the pan-caspase inhibitor Z-VAD-FMK $(50 \mu \mathrm{M})$ for $30 \mathrm{~min}$ and then treated with SR-T100 (5 $\mathrm{gg} / \mathrm{ml})$ for $12 \mathrm{~h}$. d The panel shows the percent change in the LC3-II/actin ratio compared with the untreated group in three independent experiments

risk HPV type. The Z-VAD-FMK treatment significantly increased the fold change in the LC3-II/actin ratio from 2.6 to 4.8 in Null cells, 2.2 to 5.3 in HPV6b-E6/E7 cells, and 2.8 to 4.8 in HPV11-E6/E7 cells, whereas a mild increase from 4.3 to 5.3 and 5.5 to 5.5 was observed in HPV16-E6/E7 and HPV18 -E6/E7 cells treated with SRT100, respectively (Fig. 4c). The histogram showing autophagy activity showed a fold change in the LC3-II/ actin ratio when the HPV-infected cells were treated with or without SR-T100 or the pan caspase inhibitor ZVAD-FMK, as shown in Fig. 4d.

\section{Discussion}

In this pilot study of condyloma treatment, 19 (73\%) of 26 patients using the SR-T100 gel (2.3\% solamargine in Solanum incanum plant extract) exhibited a response, and $16(61.5 \%)$ patients achieved total clearance. In addition, only one patient showed a severe (grade 3-4) skin-related side effect. Although an extract of Solanum dulcamara L. has been reported in folk medicine to treat warts [4], this is the first reported trial of the use of solamargine to treat condyloma.

External genital condyloma or genital warts (EGW) are the most common sexually transmitted diseases worldwide and are caused by HPV infection. Low-risk (LR) HPVs predominate, with a prevalence of $89 \%$, and the most prevalent genotypes are 6 and 11, followed by other high-risk (HR) HPV types [2]. Patient-applied home use medications for EGW include imiquimod, polyphenon E (green tea extracts), and podofilox (a purified podophyllin), which may produce skin erosions and burning in the majority of patients [23].

Imiquimod is an immune response modifier that has been shown to induce cytokine production (interleukins, interferon, and TNF) [24]. Podophyllotoxin, the principal active component of podophyllin, blocks microtubule assembly and inhibits the proliferation of HPV-infected 
keratinocytes [25]. However, green tea extracts have antitumor and anti-proliferative effects and induce apoptosis in cancer cells by causing G0/G1-phase cell cycle arrest or stabilizing p53 via phosphorylation on critical serine residues [26].

Treated warts were eradicated completely in $37 \%$ to $83 \%$ of patients using podofilox [27], 50\% of patients using $5 \%$ imiquimod cream [28], and 52.5 to $57.2 \%$ of patients using 15\% sinecatechin (polyphenon E) ointment [29]. The most common adverse effects of these drugs are local reactions, including erythema, pruritis/burning, pain, ulceration, and erosion, which were observed in $44 \%$ of patients using podophyllytoxin and $53.3 \%$ of patients using immiquimod [30]. In comparison, the SR-T100 gel produces good condyloma lesion responses, much less local tissue destruction and inflammation, and has the advantage over all treatments of use at home.

Oncogenic or high-risk (HR) HPV types (e.g., HPV types 16 and 18) are the cause of most cervical cancers, as well as the cause of anal, penile, vulvar, and vaginal cancers [3]. The primary transforming activity of highrisk HPVs is provided by the E6 and E7 oncoproteins. HR-HPV E6 and E7 can bind to and induce the degradation of p53 and pRB [31, 32] to exert their antiapoptotic activity. In contrast, LR-HPV E6 and E7 only bind to pRB and p53 but fail to induce degradation, exhibiting reduced anti-apoptosis ability [33, 34].

Solamargine effectively induces apoptotic cell death in various cancer cell lines, mainly by activating TNFRs $[5,7,9]$. SR-T100, which is extracted from Solanum incanum, contains alkaloid solamargine as the main active ingredient and has been show to induce cutaneous squamous cell carcinoma cell apoptosis and to inhibit tumor growth in vivo [10]. In the present study, we showed that SR-T100 induced apoptosis in HPVassociated cells in vitro and induced patient condyloma regression in a clinical trial. Cells expressing HPV6bE6/E7 and HPV11-E6/E7 were more sensitive to SRT100 treatment than those expressing HPV16-E6/E7 and HPV18-E6/E7. Our results showed that SR-T100 did not significantly increase TNF-R1 and Fas expression or caspase-8 activity in HPV-infected epithelial cells (Fig. 2a and d). SR-T100 significantly decreased MMP and caspase- 9 and -3 activity in cells expressing HPV6b-E6/E7 and HPV11-E6/E7 compared with those expressing HPV16-E6/E7 and HPV18-E6/E7, indicating that SR-T100 induced apoptosis in HPV6b- and HPV11-infected cells via the mitochondrial-dependent pathway (Fig. 2b-d). The different apoptotic responses in HPV-infected cells induced by SR-T100 in the concentration of $5 \mu \mathrm{g} / \mathrm{mL}$ may be due to differences in the anti-apoptotic activity of E6/E7 of HR or LR HPVs. Experiments with higher concentration of SR-T100 may be needed to confirm this observation.
Autophagy is an evolutionarily conserved mechanism and has been characterized as an adaptation to cell damage during stress, thus promoting cell growth and survival [21]. Autophagy and apoptosis play major roles in determining cell destiny, and the connections and cross-talk between these two processes are still being investigated. The HPV16 E7 protein was found to induce an autophagy-like process in normal human keratinocytes, which may arise as a consequence of metabolic stress [35]. However, there have been no reports regarding the difference in autophagy abilities between cells expressing LR or HR HPV E6/E7 proteins. In the present study, we first showed that the autophagy response was significantly increased in cells expressing HPV16-E6/E7 and HPV18-E6/E7 compared with those expressing HPV6b-E6/E7 and HPV11-E6/E7 after treatment with SR-T100 (Fig. 3). Because cells expressing HR HPV E6/E7 display increased anti-apoptotic activity (Fig. 1), this phenomenon may imply that cells expressing HPV16-E6/E7 or HPV18-E6/E7 exhibited increased autophagy activity to allow the cells to survive under stress conditions, i.e., treatment with SR-T100. Autophagy exerts a protective effect against apoptosis in HPV-infected cells treated with SR-T100.

Some studies have indicated that the suppression of autophagy enhances apoptosis under conditions of cellular stress [36]. In the present study, cells expressing HPV16-E6/E7 and HPV18-E6/E7 demonstrated a significant increase in apoptosis after treatment with SR-T100 using the autophagy inhibitors WT and 3MA; however, no obvious changes in apoptosis were observed in the HPV 6b-E6/E7- and HPV11-E6/E7expressing cells (Fig. 4a-b). The results obtained for cells expressing HR HPVs were consistent with previous reports [36]. Using the pan-caspase inhibitor ZVAD-FMK, we showed that cells expressing low-risk HPVs exhibited increased expression of LC3-II after treatment with SR-T100, whereas there were no obvious changes in the LC3-II levels in cells expressing high-risk HPVs (Fig. 4c-d). Because autophagy-related genes (ATGs), i.e., ATG6 and ATG3, have been reported to be caspase substrates [37, 38] and Z-VADFMK can increase LC3-II formation [39], cells treated with Z-VAD-FMK may display increased autophagy activities. The lack of an obvious change in LC3-II expression in cells expressing HR HPVs may be because LC3-II reached the maximum level of autophagy, as previously reported [40].

Our results suggested that autophagy has a protective function in HPV-infected cells, particularly in SR-T100treated cells infected with high-risk type HPVs. However, the detailed mechanism of SR-T100-induced apoptosis and autophagy in HPV-infected cells requires furthers investigation. 


\section{Conclusion}

Our pilot study showed that SR-T100 was effective for the treatment of human vulva condyloma and was associated with few side effects. Furthermore, we showed that cells expressing LR HPVs were more sensitive to SR-T100 treatment than those expressing HR HPVs. We suggest that autophagy is involved and plays a protective in SR-T100-induced apoptosis in HPV-infected cells. Further clinical trials are needed to evaluate its effectiveness for the treatment of condyloma.

\section{Additional file}

Additional file 1: Table S1. Protocol and treatment flow chart. Table S2. Primers sequences for polymerase chain reactions. Figure S1. HPLC Fingerprint of SR-T100. Figure S2. Apoptosis inhibition by Z-VAD-FMK significantly recovered cell viability in cells infected with low-risk type HPVs. (DOCX 285 kb)

\section{Abbreviations}

HPV: Human papillomaviruses; MMP: Mitochondrial membrane potential; TEM: Transmission electron microscopy

\section{Acknowledgments}

We are grateful to the RNAi Core Lab, Research Center of Clinical Medicine, National Cheng Kung University Hospital, Taiwan for producing the lentiviral particles. We thank Dr. Sheng-Hsiang Lin in the Biostatistics Consulting Center, National Cheng Kung University Hospital for providing the statistical consultation. We thank Ms. Shing-Chih Tsai in the Department of Pathology, National Cheng Kung University Hospital for assistance with electron microscopy.

\section{Funding}

This work was supported by grants from the National Cheng Kung University Hospital (NCKUH-10306004) and the Ministry of Science and Technology of Taiwan (NSC 101-2325-B-006 -015 and MOST 104-2314-B-006 -072 -MY2).

\section{Availability of data and materials}

All data generated or analysed during this study are included in this published article and its supplementary information files.

\section{Authors' contributions}

CYC and HKF conducted and coordinated the experiments, analyzed the data and drafted the manuscript. SMR, LYL and HKF acquired and managed patients. SAL provided the material. SHM discussed the experiments and the manuscript. CYC and HKF coordinated and discussed the experiments and revised the manuscript. All authors read and approved the final version of the manuscript.

\section{Ethics approval and consent to participate}

This study approved by the Institutional Review Board at National Cheng Kung University Hospital (Tainan, Taiwan) (IRB No:HR-98-069) and informed consent from the patients.

\section{Consent for publication}

Not applicable (no patient identifier or personalized data shown).

\section{Competing interests}

The authors declare that they have no competing interests.

\section{Publisher's Note}

Springer Nature remains neutral with regard to jurisdictional claims in published maps and institutional affiliations.

\section{Author details}

${ }^{1}$ Department of Obstetrics and Gynecology, National Cheng Kung University Hospital, College of Medicine, National Cheng Kung University, 138 Sheng Li
Road, Tainan 704, Taiwan. ${ }^{2}$ Microbiology and Immunology, National Cheng Kung University Hospital, College of Medicine, National Cheng Kung University, Tainan, Taiwan. ${ }^{3}$ Dermatology, National Cheng Kung University Hospital, College of Medicine, National Cheng Kung University, Tainan, Taiwan.

Received: 21 February 2017 Accepted: 3 November 2017

Published online: 10 November 2017

\section{References}

1. Workowski KA, Bolan GA. Centers for disease C, prevention. Sexually transmitted diseases treatment guidelines, 2015. MMWR Recomm Rep. 2015;64:1-137.

2. Aubin F, Pretet $J$, Jacquard $A C$, Saunier $M$, Carcopino $X$, Jaroud $F$, Pradat $P$, Soubeyrand B, Leocmach Y, Mougin C, et al. Human papillomavirus genotype distribution in external acuminata condylomata: a large French National Study (EDiTH IV). Clin Infect Dis. 2008;47:610-5.

3. Ghittoni R, Accardi R, Hasan U, Gheit T, Sylla B, Tommasino M. The biological properties of E6 and E7 oncoproteins from human papillomaviruses. Virus Genes. 2010;40:1-13.

4. Kupchan SM, Barboutis SJ, Knox JR, Cam CA. Beta-solamarine: tumor inhibitor isolated from Solanum Dulcamara. Science. 1965;150:1827-8.

5. Liu LF, Liang CH, Shiu LY, Lin WL, Lin CC, Kuo KW. Action of solamargine on human lung cancer cells-enhancement of the susceptibility of cancer cells to TNFs. FEBS Lett. 2004;577:67-74.

6. Kuo KW, Hsu SH, Li YP, Lin WL, Liu LF, Chang LC, Lin CC, Lin CN, Sheu HM. Anticancer activity evaluation of the solanum glycoalkaloid solamargine. Triggering apoptosis in human hepatoma cells. Biochem Pharmacol. 2000;60:1865-73.

7. Hsu SH, Tsai TR, Lin CN, Yen MH, Kuo KW. Solamargine purified from Solanum Incanum Chinese herb triggers gene expression of human TNFR I which may lead to cell apoptosis. Biochem Biophys Res Commun. 1996;229:1-5.

8. Liang CH, Shiu LY, Chang LC, Sheu HM, Kuo KW. Solamargine upregulation of Fas, downregulation of HER2, and enhancement of cytotoxicity using epirubicin in NSCLC cells. Mol Nutr Food Res. 2007;51:999-1005.

9. Shiu LY, Chang LC, Liang CH, Huang YS, Sheu HM, Kuo KW. Solamargine induces apoptosis and sensitizes breast cancer cells to cisplatin. Food Chem Toxicol. 2007:45:2155-64.

10. CH W, Liang CH, Shiu LY, Chang LC, Lin TS, Lan CC, Tsai JC, Wong TW, Wei KJ, Lin TK, et al. Solanum Incanum extract (SR-T100) induces human cutaneous squamous cell carcinoma apoptosis through modulating tumor necrosis factor receptor signaling pathway. J Dermatol Sci. 2011;63:83-92.

11. YH W, Chiu WT, Young MJ, Chang TH, Huang YF, Chou CY. Solanum Incanum extract Downregulates Aldehyde Dehydrogenase 1-mediated Stemness and inhibits tumor formation in ovarian cancer cells. J Cancer. 2015;6:1011-9.

12. Hsu KF, CL W, Huang SC, Hsieh JL, Huang YS, Chen YF, Shen MR, Chung WJ, Chou CY, Shiau AL. Conditionally replicating E1B-deleted adenovirus driven by the squamous cell carcinoma antigen 2 promoter for uterine cervical cancer therapy. Cancer Gene Ther. 2008;15:526-34.

13. Lin CN, CM L, Cheng MK, Gan KH, Won SJ. The cytotoxic principles of Solanum Incanum. J Nat Prod. 1990;53:513-6.

14. Hsu KF, Huang SC, Shiau AL, Cheng YM, Shen MR, Chen YF, Lin CY, Lee BH, Chou CY. Increased expression level of squamous cell carcinoma antigen 2 and 1 ratio is associated with poor prognosis in early-stage uterine cervical cancer. Int J Gynecol Cancer. 2007;17:174-81.

15. Munoz N, Bosch FX, de Sanjose S, Herrero R, Castellsague X, Shah KV, Snijders PJ, Meijer CJ. International Agency for Research on Cancer multicenter cervical cancer study G. Epidemiologic classification of human papillomavirus types associated with cervical cancer. N Engl J Med. 2003;348:518-27.

16. Shiau AL, Teo ML, Chen SY, Wang CR, Hsieh JL, Chang MY, Chang CJ, Chao J, Chao L, CL W, Lee CH. Inhibition of experimental lung metastasis by systemic lentiviral delivery of kallistatin. BMC Cancer. 2010;10:245.

17. BH S, Tseng YL, Shieh GS, Chen YC, Shiang YC, Wu P, Li KJ, Yen TH, Shiau $\mathrm{AL}, \mathrm{Wu} \mathrm{CL}$. Prothymosin alpha overexpression contributes to the development of pulmonary emphysema. Nat Commun. 2013, 1906;4

18. Hsu KF, CL W, Huang SC, CM W, Hsiao JR, Yo YT, Chen YH, Shiau AL, Chou $C Y$. Cathepsin L mediates resveratrol-induced autophagy and apoptotic cell death in cervical cancer cells. Autophagy. 2009;5:451-60.

19. Yo YT, Shieh GS, Hsu KF, CL W, Shiau AL. Licorice and licochalcone-a induce autophagy in LNCaP prostate cancer cells by suppression of $\mathrm{BCl}-2$ expression and the mTOR pathway. J Agric Food Chem. 2009;57:8266-73. 
20. Klionsky DJ, Abdelmohsen K, Abe A, Abedin MJ, Abeliovich H, Acevedo Arozena A, Adachi H, Adams CM, Adams PD, Adeli K, et al. Guidelines for the use and interpretation of assays for monitoring autophagy (3rd edition). Autophagy. 2016;12:1-222.

21. Ferraro E, Cecconi F. Autophagic and apoptotic response to stress signals in mammalian cells. Arch Biochem Biophys. 2007:462:210-9.

22. Kabeya Y, Mizushima N, Ueno T, Yamamoto A, Kirisako T, Noda T, Kominami E, Ohsumi Y, Yoshimori T. LC3, a mammalian homologue of yeast Apg8p, is localized in autophagosome membranes after processing. EMBO J. 2000;19:5720-8,

23. Kollipara R, Ekhlassi E, Downing C, Guidry J, Lee M, Tyring SK. Advancements in pharmacotherapy for noncancerous manifestations of HPV. J Clin Med. 2015:4:832-46.

24. Reiter MJ, Testerman TL, Miller RL, Weeks CE, Tomai MA. Cytokine induction in mice by the immunomodulator imiquimod. J Leukoc Biol. 1994:55:234-40.

25. Lacey CJ, Goodall RL, Tennvall GR, Maw R, Kinghorn GR, Fisk PG, Barton S, Byren I. Perstop Pharma genital warts clinical trial G. Randomised controlled trial and economic evaluation of podophyllotoxin solution, podophyllotoxin cream, and podophyllin in the treatment of genital warts. Sex Transm Infect. 2003; $79: 270-5$

26. Stockfleth $E$, Meyer $T$. The use of sinecatechins (polyphenon E) ointment for treatment of external genital warts. Expert Opin Biol Ther. 2012;12:783-93.

27. Stone KM. Human papillomavirus infection and genital warts: update on epidemiology and treatment. Clin Infect Dis. 1995;20(Suppl 1):S91-7.

28. Edwards L, Ferenczy A, Eron L, Baker D, Owens ML, Fox TL, Hougham AJ, Schmitt KA. Self-administered topical 5\% imiquimod cream for external anogenital warts. HPV study group. Human PapillomaVirus. Arch Dermatol. 1998;134:25-30.

29. Tatti S, Swinehart JM, Thielert C, Tawfik H, Mescheder A, Beutner KR. Sinecatechins, a defined green tea extract, in the treatment of external anogenital warts: a randomized controlled trial. Obstet Gynecol. 2008;111:1371-9.

30. Komericki P, Akkilic-Materna M, Strimitzer T, Aberer W. Efficacy and safety of imiquimod versus podophyllotoxin in the treatment of anogenital warts. Sex Transm Dis. 2011:38:216-8.

31. Dyson N, Howley PM, Munger K, Harlow E. The human papilloma virus-16 E7 oncoprotein is able to bind to the retinoblastoma gene product. Science. 1989;243:934-7.

32. Werness BA, Levine AJ, Howley PM. Association of human papillomavirus types 16 and 18 E6 proteins with p53. Science. 1990;248:76-9.

33. Gage JR, Meyers C, Wettstein FO. The E7 proteins of the nononcogenic human papillomavirus type $6 \mathrm{~b}$ (HPV-6b) and of the oncogenic HPV-16 differ in retinoblastoma protein binding and other properties. J Virol. 1990;64:723-30.

34. Boyer SN, Wazer DE, Band V. E7 protein of human papilloma virus-16 induces degradation of retinoblastoma protein through the ubiquitinproteasome pathway. Cancer Res. 1996;56:4620-4.

35. Zhou X, Munger K. Expression of the human papillomavirus type 16 E7 oncoprotein induces an autophagy-related process and sensitizes normal human keratinocytes to cell death in response to growth factor deprivation. Virology. 2009;385:192-7.

36. Boya P, Gonzalez-Polo RA, Casares N, Perfettini JL, Dessen P, Larochette N, Metivier D, Meley D, Souquere S, Yoshimori T, et al. Inhibition of macroautophagy triggers apoptosis. Mol Cell Biol. 2005;25:1025-40.

37. Cho DH, Jo YK, Hwang JJ, Lee YM, Roh SA, Kim JC. Caspase-mediated cleavage of ATG6/Beclin-1 links apoptosis to autophagy in HeLa cells. Cancer Lett. 2009;274:95-100.

38. Oral O, Oz-Arslan D, Itah Z, Naghavi A, Deveci R, Karacali S, Gozuacik D. Cleavage of Atg3 protein by caspase-8 regulates autophagy during receptor-activated cell death. Apoptosis. 2012;17:810-20.

39. Herzog C, Yang C, Holmes A, Kaushal GP. ZVAD-fmk prevents cisplatininduced cleavage of autophagy proteins but impairs autophagic flux and worsens renal function. Am J Physiol Renal Physiol. 2012;303:F1239-50.

40. Li X, Su J, Xia M, Li H, Xu Y, Ma C, Ma L, Kang J, Yu H, Zhang Z, Sun L. Caspase-mediated cleavage of Beclin1 inhibits autophagy and promotes apoptosis induced by 51 in human ovarian cancer SKOV3 cells. Apoptosis. 2016:21:225-38.

\section{Submit your next manuscript to BioMed Central and we will help you at every step:}

- We accept pre-submission inquiries

- Our selector tool helps you to find the most relevant journal

- We provide round the clock customer support

- Convenient online submission

- Thorough peer review

- Inclusion in PubMed and all major indexing services

- Maximum visibility for your research

Submit your manuscript at www.biomedcentral.com/submit

) Biomed Central 